Article

\title{
Detection and Segmentation of Small Trees in the Forest-Tundra Ecotone Using Airborne Laser Scanning
}

\author{
Marius Hauglin * and Erik Næsset \\ Norwegian University of Life Sciences, Department of Ecology and Natural Resource Management, \\ P.O. Box 5003, N-1432 Ås, Norway; erik.naesset@nmbu.no \\ * Correspondence: marius.hauglin@nmbu.no; Tel.: +47-672-316-89 \\ Academic Editors: Jie Shan, Juha Hyyppä, Lars T. Waser and Prasad S. Thenkabail \\ Received: 10 December 2015; Accepted: 4 May 2016; Published: 11 May 2016
}

\begin{abstract}
Due to expected climate change and increased focus on forests as a potential carbon sink, it is of interest to map and monitor even marginal forests where trees exist close to their tolerance limits, such as small pioneer trees in the forest-tundra ecotone. Such small trees might indicate tree line migrations and expansion of the forests into treeless areas. Airborne laser scanning (ALS) has been suggested and tested as a tool for this purpose and in the present study a novel procedure for identification and segmentation of small trees is proposed. The study was carried out in the Rollag municipality in southeastern Norway, where ALS data and field measurements of individual trees were acquired. The point density of the ALS data was eight points per $\mathrm{m}^{2}$, and the field tree heights ranged from 0.04 to $6.3 \mathrm{~m}$, with a mean of $1.4 \mathrm{~m}$. The proposed method is based on an allometric model relating field-measured tree height to crown diameter, and another model relating field-measured tree height to ALS-derived height. These models are calibrated with local field data. Using these simple models, every positive above-ground height derived from the ALS data can be related to a crown diameter, and by assuming a circular crown shape, this crown diameter can be extended to a crown segment. Applying this model to all ALS echoes with a positive above-ground height value yields an initial map of possible circular crown segments. The final crown segments were then derived by applying a set of simple rules to this initial "map" of segments. The resulting segments were validated by comparison with field-measured crown segments. Overall, $46 \%$ of the field-measured trees were successfully detected. The detection rate increased with tree size. For trees with height $>3 \mathrm{~m}$ the detection rate was $80 \%$. The relatively large detection errors were partly due to the inherent limitations in the ALS data; a substantial fraction of the smaller trees was hit by no or just a few laser pulses. This prevents reliable detection of changes at an individual tree level, but monitoring changes on an area level could be a possible application of the method. The results further showed that some variation must be expected when the method is used for repeated measurements, but no significant differences in the mean number of segmented trees were found over an intensively measured test area of 11.4 ha.
\end{abstract}

Keywords: airborne laser scanning; treeline; monitoring

\section{Introduction}

Airborne laser scanning (ALS) is used today as a tool for forest applications, both for research purposes as well as in operational settings. Productive forest has in many places been the main target, but ALS can also be applied in other types of forest. The very frontiers of the forests have in many places gradually expanded into alpine areas [1], and this expansion is believed to be caused by several factors, with reduced grazing by domestic livestock and climate changes as two dominating causes. 
Expansion of forests into areas such as the forest-tundra ecotone will influence carbon sequestration, but will also in many places have a direct effect on the climate through the so-called "albedo-effect". The darker-colored trees will reflect less of the solar radiation than bare ground, especially in the winter when the ground is covered with snow. The net effect of this phenomenon is warming [2].

It is therefore of interest to map and monitor possible changes taking place in the vegetation structure of the forest-tundra ecotone, such as the appearance of pioneer trees and the migration of the tree line. ALS has through several studies been proposed and tested as a tool for this task [3-8]. Several of these studies investigate and document the potential for discriminating between echoes reflected from trees and echoes reflected from other objects using metrics derived from ALS data, such as the height above the modeled terrain surface, the backscatter intensity of the echoes and the properties of the spatial distribution of echoes. There is, however, a need to apply this knowledge and further develop methods to derive quantitative properties such as tree numbers, crown coverage or tree size distributions to enable the establishment of efficient monitoring methodologies. A segmentation and identification of single trees from the ALS data would be one possible way of deriving such properties. Numerous studies have already proposed and tested methods to derive single-tree segments from ALS data [9-13]. An introduction to tree segmentation and an overview of these methods can be found in Koch et al. [14]. Common to all of these studies are that they are focused on mature forests, with an emphasis on trees considerably larger than those typically found in the forest-tundra ecotone. We did not consider any of the described methods to be directly applicable to the task of deriving single-tree information for smaller trees in the forest-tundra ecotone because with an average point density of, for example, 5-10 points per $\mathrm{m}^{2}$, the number of echoes from each individual tree will typically range from one single echo up to less than 100 in most cases.

Many of the existing segmentation methods involve interpolation of the ALS point cloud to a raster or to a three-dimensional voxel space [14]. However, such methods typically assume a choice of a fixed pixel—or voxel—size. This pixel size will be closely linked to the range of tree sizes which can be detected. A large pixel size will smooth out the information inherent in the ALS point cloud and therefore make the detection of small trees harder, whereas smaller pixels will likely cause over-segmentation of echoes from larger trees. With these existing methods one is, in practice, faced with a choice of detecting trees within a limited size range, through the choice of a fixed pixel size. The chosen pixel size and the level of smoothing applied will also determine the spatial extent of each segmented tree crown. The extent of the segmented tree crowns is typically represented by pixels, which could limit the ability to accurately represent the crown of small trees. We wanted a method that could detect trees ranging from small to medium in size, and rather than modifying any of the existing methods, we developed a simple and novel segmentation procedure. Thus, the proposed segmentation procedure was specifically tailored to the detection of small trees, with as few as only one laser echo. It should be noted that its area of application could be wider than just small trees in the forest-tundra ecotone, including, for example, the monitoring of seedlings in forest stands planted after final fellings in managed boreal forests, or the detection and monitoring of small trees in afforested areas in the tropics.

The objectives of the present study were (1) to develop a procedure for automatic detection and segmentation of small trees using ALS data and (2) to assess the accuracy of the method by comparing the results with field reference data. We consider small trees in this context to be trees with heights up to $7 \mathrm{~m}$ and crown diameters up to $6 \mathrm{~m}$. We further wanted to assess the suitability for monitoring purposes by testing the stability of the method across two separate ALS acquisitions for the same study area.

\section{Materials and Methods}

\subsection{Study Area}

The study area is located in the Rollag municipality in southeastern Norway $\left(60^{\circ} 0^{\prime} \mathrm{N} 9^{\circ} 01^{\prime} \mathrm{E}\right.$, 910-950 m above sea level) and is constituted by a rectangle of $200 \times 600 \mathrm{~m}$ centered on a mountain ridge. The data materials used were from registrations in the tree line which, at this location, is around 
900-940 m above sea level (Figure 1). The main tree species are downy birch (Betula pubescens ssp czerepanovii), Scots pine (Pinus sylvestris L.) and Norway spruce (Picea abies (L.) Karst.).

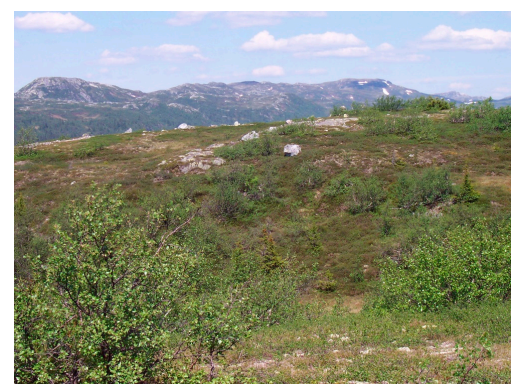

Figure 1. Picture of the landscape and vegetation in the study area.

\subsection{Field Data}

Field registrations from 472 positioned trees ranging from 0.04 to $6.3 \mathrm{~m}$ in height were used in the present study (Table 1). The field work was conducted during the summer of 2012. Within the study area, 40 points were systematically laid out, and at each point up to 16 trees were selected according to the so-called point-centered quarter sampling method sampling procedure [15]: At each point a circular area with a radius of $25 \mathrm{~m}$ was sectioned into four quadrants along the cardinal directions $\mathrm{N}-\mathrm{S}$ and E-W. Using four height classes $(0-1,1-2,2-3$ and $>3 \mathrm{~m})$, the tree nearest to the center point in each class and quadrant was selected, giving a maximum of four sample trees per quadrant. The purpose of the procedure was to establish a consistent method to sample trees across the entire range of tree heights found in the area.

Table 1. Summary of the field-measured trees.

\begin{tabular}{cccc}
\hline & Height $(\mathbf{m})$ & Mean Crown Diameter $(\mathbf{m})$ & $\mathbf{n}$ \\
\hline & min-max (mean) & min-max (mean) \\
\hline Deciduous trees $^{\text {a }}$ & $0.04-6.30(1.73)$ & $0.03-5.50(1.37)$ & 193 \\
Pine & $0.06-1.99(0.47)$ & $0.03-1.35(0.37)$ & 83 \\
Spruce & $0.05-3.08(1.49)$ & $0.07-3.35(1.28)$ & 196 \\
All & $0.04-6.30(1.41)$ & $0.03-5.50(1.15)$ & 472 \\
\hline \multicolumn{4}{c}{ a Mainly birch. }
\end{tabular}

Each of the sample trees were positioned with real-time differential Global Navigation Satellite Systems, with an expected accuracy of 3-4 cm. For each tree, the species, height and horizontal crown diameter in the N-S and E-W directions were recorded. The height and crown diameters were recorded with a measuring tape, with the heights of the highest trees recorded with a Haglöfs Vertex III hypsometer.

The horizontal extent-or crown projection-of all field-measured trees in the data material was defined as an ellipse created from the tree position and the two perpendicular crown diameter measurements. These field-measured crown segments were used when extracting ALS echoes from individual trees, and as a field reference in the validation.

\subsection{ALS Data}

Two sets of ALS data were used in the present study. The first set of ALS data was acquired in July 2006 with an Optech ALTM 3100 laser scanner mounted on a fixed-wing aircraft. These data were acquired in two overlapping flight lines, which means that parts of the study area were covered by ALS data from both flight lines. This dataset was used to test the stability of the proposed segmentation procedure (further described in Section 2.4). 
The second set of ALS data was acquired in August 2012 with a Leica ALS70 laser scanner mounted on a fixed-wing aircraft. This dataset corresponded in time with the field registrations and was used to develop and test the segmentation procedure. The two datasets are denoted ALS2006 and ALS2012 throughout this paper. Further details of the ALS2006 and ALS2012 datasets are given in Table 2.

Table 2. Specifications of the two ALS datasets. Mean echo and pulse density calculated from the data, information in the other fields from the data vendor.

\begin{tabular}{ccc}
\hline Dataset & ALS2006 & ALS2012 \\
\hline Sensor & Optech ALTM3100 & Leica ALS70 \\
Scan frequency (Hz) & 70 & - \\
Pulse frequency (kHz) & 100 & 154.4 \\
Flying speed (m/s) & 75 & 69 \\
Mean flying altitude (a.g.1.) & $800 \mathrm{~m}$ & $1800 \mathrm{~m}$ \\
Mean point density (echoes per $\left.\mathrm{m}^{2}\right)$ & 8 & 15 \\
Mean pulse density (pulses per $\left.\mathrm{m}^{2}\right)$ & 8 & 15 \\
Footprint diameter $(\mathrm{m})$ & 0.21 & 0.27 \\
Vertical accuracy $(\mathrm{m})$ & 0.10 & 0.12 \\
Planimetric accuracy $(\mathrm{m})$ & 0.13 & 0.20 \\
Maximum iteration angle (degrees) & 9 & 7.5 \\
Maximum iteration distance $(\mathrm{m})$ & 1 & 1.9 \\
\hline
\end{tabular}

First return echoes were used from the ALS2006 dataset, and all returns from the ALS2012 dataset. Note that-with respect to return categories-the difference between the two datasets was small, since most pulses yield only a single echo from the generally low vegetation in the study area. We found that approximately $97 \%$ of the echoes in the ALS2012 dataset were, in fact, single returns. The positional accuracy of the laser echoes was expected to be in the range of $0.1-0.2 \mathrm{~m}$ for both sensors, according to Ussyshkin and Smith [16] and the Leica ALS70 product brochure [17].

The ALS echoes were, for both datasets, classified into ground and non-ground using the Terrascan software, following the triangular irregular network (TIN) densification algorithm described by Axelsson [18]. Control parameters for the ground classification, the so-called "maximum iteration angle" and "maximum iteration distance", were set to 9 degrees and $1 \mathrm{~m}$ for the first ALS dataset. In the second dataset, values of 7.5 degrees and $1.9 \mathrm{~m}$ were used (see [19]). Above-ground heights were calculated for all echoes, as the distance between the TIN and the recorded ellipsoidal heights.

A selection of the ALS and field data is visualized in Figure 2.

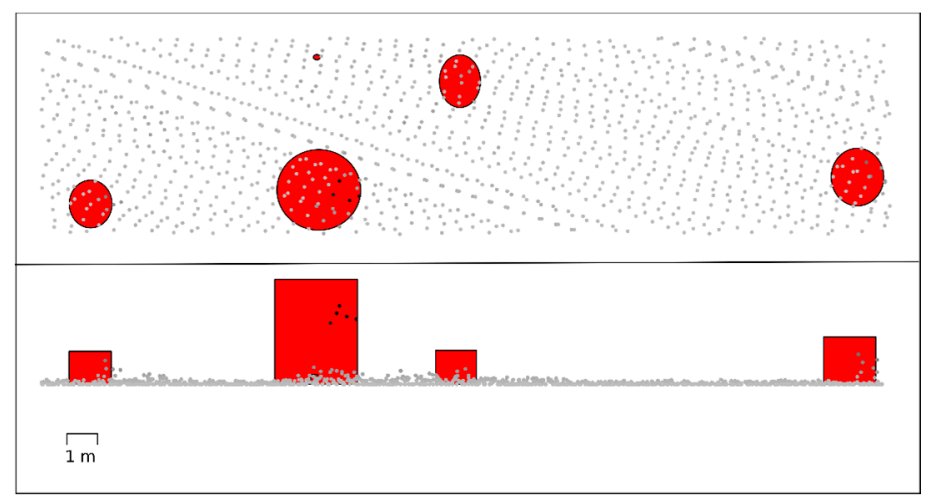

Figure 2. Visualization of five field-measured trees, and the ALS echoes from ALS2012 in the corresponding area. Viewed from above (upper figure) and from the side (lower figure). The fieldmeasured height and crown extent is colored red, and the ALS echoes are colored from grey to black. The highest echoes are colored black. Note that the terrain height has been subtracted from the ALS echo heights (see text for details), and that only a sample of trees was measured. 


\subsection{Calculations and Analysis}

The field dataset was split in to a modeling dataset consisting of four of the 40 sample locations (locations \#10, \#20, \#30 and \#40), and a validation dataset consisting of the remaining 36 locations. This resulted in a set of 39 trees for modeling, and a set of 433 trees for validation. Note that the validation dataset was used to validate the whole segmentation process, whereas the modeling data were used for the two models described in the following. The rationale for this split of the data was to have a sufficient number of trees for the two allometric models, but with an emphasis on the validation of the whole segmentation process. We considered the chosen number of trees in the modeling dataset to be sufficient for the two simple linear models. A summary of the model and validation datasets is given in Table 3.

Table 3. Number of trees in the modeling and validation datasets.

\begin{tabular}{ccc}
\hline Height Class & Model Dataset & Validation Dataset \\
\hline $0-1 \mathrm{~m}$ & $18(46 \%)$ & $202(47 \%)$ \\
$1-2 \mathrm{~m}$ & $11(28 \%)$ & $119(27 \%)$ \\
$2-3 \mathrm{~m}$ & $6(16 \%)$ & $61(14 \%)$ \\
$>3 \mathrm{~m}$ & $4(10 \%)$ & $51(12 \%)$ \\
all & 39 & 433 \\
\hline
\end{tabular}

The proposed method is based on two models, one allometric model relating field-measured tree height to crown diameter, and one model relating field-measured tree height to the above-ground height of the ALS echoes.

\subsubsection{Height-Crown Diameter Model}

The field registrations in the modeling data were used to fit a non-intercept linear regression model relating crown diameter to tree height

$$
\widehat{c d}=\beta_{a} \cdot h+\epsilon_{a}
$$

where $\hat{c d}$ is the crown diameter defined as the mean of the two perpendicular crown diameter measurements, $h$ is the field-measured tree height, $\beta_{a}$ is the parameter to be estimated and $\epsilon_{a}$ is an error term, expected to be normally distributed with mean zero. Other model forms and transformations of the variables were tested, but did not result in substantially better models with this data. The simplest linear model was used in the present study, and other models are not further documented. A non-intercept model was chosen in order to ensure positive predictions of $c d$ for all $h>0$, and to satisfy the condition that $c d=0$ when $h=0$. The coefficient of determination for the non-intercept model was calculated as the square of the Pearson's correlation of the fitted and observed values.

The relationship between height and crown diameter might vary between species, but since species information cannot easily be obtained from ALS data we could not use species-specific models for predictions. The species information were therefore not used when we fitted the model given by Equation (1).

\subsubsection{ALS Echo Height-Field-Measured Height Model}

The crown segments formed from the field-measured crown diameters were used to extract echoes from the ALS2012 dataset for each tree. All echoes inside the crown segment were assigned to the tree for which the segment was created. We did not introduce specific procedures for handling overlapping crowns, which means that a single echo could theoretically be assigned to more than one tree. It further means that any given echo assigned to a tree could have been reflected from an overlapping part of another tree. In the modeling data the maximum above-ground height of the echoes assigned to a tree was denoted $\operatorname{mmax}_{A L S}$ and related to the field-measured tree height through a linear regression model 


$$
\hat{h}=\beta_{b 0}+\beta_{b 1} \cdot \operatorname{hmax}_{A L S}+\epsilon_{b}
$$

where $\hat{h}$ is the estimated tree height, $\beta_{b 0}$ and $\beta_{b 1}$ are parameters to be estimated and $\epsilon_{b}$ is an error term.

\subsubsection{Model Fit and Validation}

Model fit was assessed by inspecting the coefficient of determination, and the models were further validated through a leave-one-out cross-validation procedure. Root mean squared error (RMSE) was computed as

$$
\operatorname{RMSE}=\sqrt{\frac{\sum_{\mathrm{i}=1}^{n}\left(\mathrm{x}_{i}-\hat{x}_{i}\right)^{2}}{n}}
$$

where $n$ is the number of trees, $x_{i}$ is the reference value of the $i$ th tree and $\hat{x}_{i}$ is the corresponding value predicted by the model constructed from the remaining $n-1$ trees in the cross-validation. We denoted RMSE as a percentage of the mean reference value as RMSE\%.

\subsubsection{Crown Segments}

Using the two described models (Equations (1) and (2)), every individual echo with a positive above-ground height was related to a circular crown segment, positioned with the given echo in its center (Figures 3 and 4). Equation (2) was used to estimate a tree height from the above-ground height of the ALS echo, and then this tree height was used in Equation (1) to get an estimated crown width, and thereby produce an initial circular crown segment. Thus, at this initial stage all echoes with a positive above-ground height were related to a positioned crown segment. The final crown segments were then determined by applying a set of simple rules to the initial crown segments:

- All echoes that fell within the circular crown segment of an echo higher above ground (i.e., a larger segment as per the height-crown diameter model) were assumed to belong to the larger segment, and such smaller segments were therefore removed before the subsequent steps. This procedure was carried out according to segment size, so that echoes within larger segments were removed first.

- In the next step, overlapping segments were identified and, based on the degree of overlap, the two underlying echoes were either assumed to be from different trees and the corresponding segments kept separate, or they were assumed to be reflected from the same tree. If the latter was true, the smaller segment was merged with the larger. Segments with an overlapping part of the two radii of more than $s$ times the smaller radius were merged. In the case of merging two segments, the lowest echo was added as a new vertex in the largest segment (Figures 3 and 4). In the present study we tested values of $s$ between 0.05 and 0.85 .

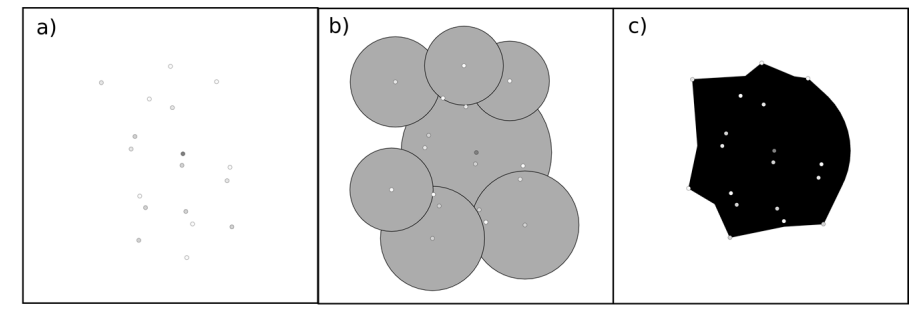

Figure 3. Graphical representation of steps in the segmentation procedure: (a) Laser echoes viewed from above, darker color indicates echoes higher above ground; (b) Each echo is associated with a circular segment. Note that the segment of the echo highest above ground is created first, and echoes inside this segment are treated as reflected from this segment; (c) Overlapping circular segments are merged based on the degree of overlap (see text for details), with the echoes of the smaller segments added as vertices in the larger segment, forming the final segment (shown in black). 
The procedure described in this section was implemented in the programming language $R$ [20] and $\mathrm{C}++$ as a fully automated algorithm.

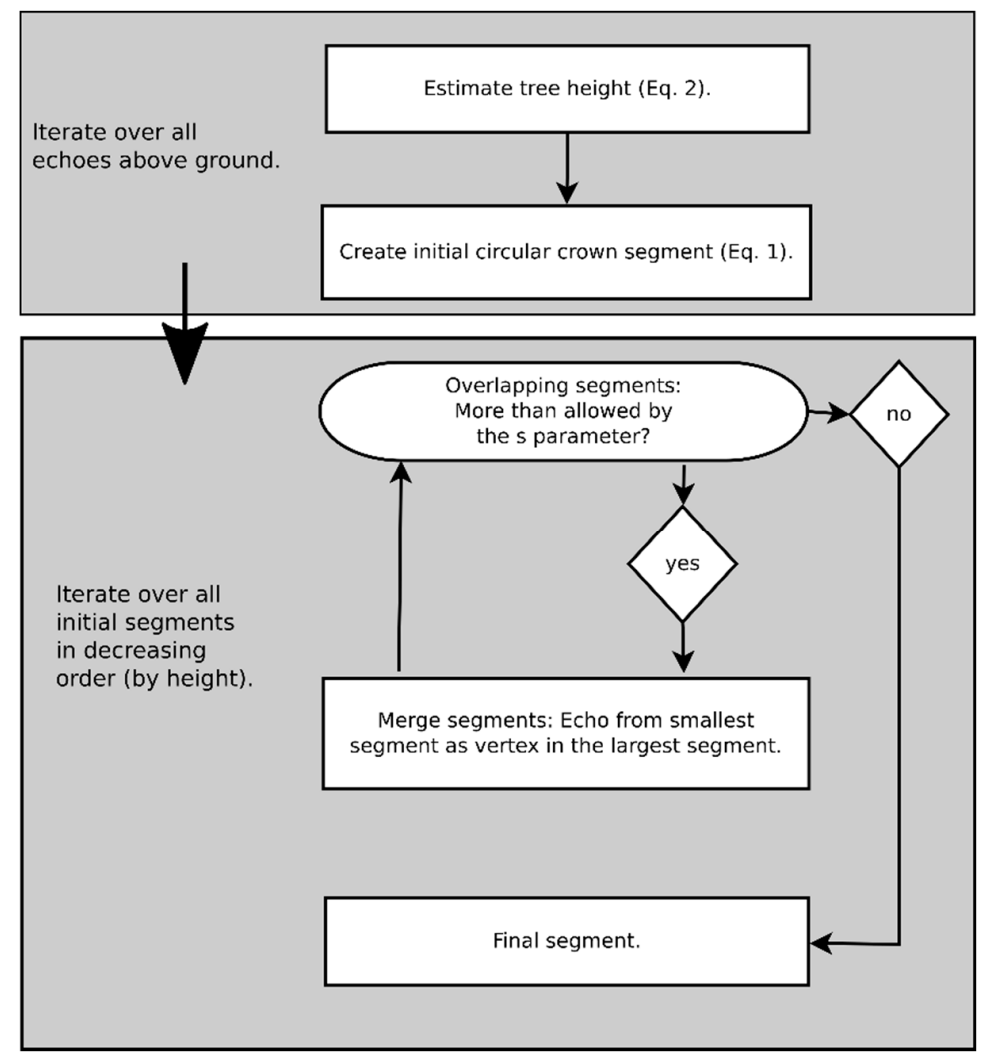

Figure 4. Flow diagram showing an outline of the segmentation process.

\subsubsection{Validation}

The ALS-derived crown segments were compared to the field-measured crown segments, and the number of field-measured segments matched by an ALS-derived segment was noted. A "match" in this context is not universally defined, so we had to rely on a set of criteria to decide if two segments matched up or not. The set of criteria we used basically defines the degree of similarity in terms of size and position that is required to label a field-measured segment as matched by an ALS-derived segment, thus regarding the tree as detected and correctly segmented.

An ALS-derived segment was defined to be successfully matched with a field tree by using the following procedure:

Each field-measured tree was linked to an ALS-derived tree if the position of the ALS-derived tree was inside the field-measured crown segment. If more than one ALS-derived tree was inside the field-measured crown segment, the ALS-derived tree with the smallest planar distance to the field tree position was used. The location of the highest ALS echo within the ALS-derived segment was used as the ALS-derived tree position.

Linked pairs of trees with large differences in height were excluded. This was done by fitting a regression model $\hat{h} \sim h$, and excluding all pairs of linked trees with a height difference larger than two times the standard error of the model.

This procedure corresponds to the procedure used in a comparison of segmentation algorithms by Vauhkonen et al. [21].

We calculated detection rates for the individual height classes described in Section 2.2 as the number of correctly segmented field trees in the particular height class to the total number field trees in that class. 


\subsubsection{Stability—Number of Segmented Trees}

Due to the design of the field work, we could not calculate the commission error. We did, however, test the stability of the number of ALS-segmented trees between two separate ALS acquisitions within a given area. In other words, we tested-for different height classes-if a similar number of trees would be segmented from a separate, second acquisition of ALS data. One aim of the current project was to develop a method that was suitable for monitoring, or repeated measurements. In such a monitoring approach it is desirable to have ALS-derived metrics which are stable, i.e., which vary little due to properties of the scanning and segmentation process itself. Thus, as much as possible of the variation between similar metrics derived from two separate acquisitions should ideally stem from actual changes in the vegetation.

The ALS2006 dataset was used, with two acquisitions carried out on the same day. The same sensor and flight parameters were used for the two acquisitions. The study area was divided into hexagonal cells of $200 \mathrm{~m}^{2}$ and after application of the proposed segmentation algorithm, the number of segmented trees from within each cell was counted. This was done separately with data from each of the two ALS acquisitions. Note that we, in the segmentation procedure here, used the existing models derived by Equations (1) and (2) and the field and ALS data from 2012.

Differences in the number of segmented trees between the two acquisitions were tested by fitting linear mixed effects models, described further in this section. The use of this approach was motivated by the ability to incorporate assumptions about spatial correlation in the test procedure. We asserted that the observations might be spatially correlated, which would violate the assumption of independent observations in statistical tests such as a paired $t$-test. Following the approach described by Zuur et al. [22] and Pinheiro and Bates [23], we tested the difference between the mean number of trees in each height class by fitting a linear mixed effects model:

$$
y_{i}=x_{i} \beta+b_{i}+\epsilon_{i}, \quad i=1, \ldots, M
$$

where $y_{i}$ is a vector with the number of segmented trees in cell $i$ and $x_{i}$ is a vector of the corresponding acquisitions as factors. We derived the number of trees from two different acquisitions, so $y_{i}$ and $x_{i}$ will be vectors of length two. Then $\boldsymbol{\beta}$ is a vector of the regression model parameters (fixed effects), $\boldsymbol{b}_{i}$ is a vector of random effects allowed to differ for each cell, $\epsilon_{i}$ is an error vector and $M$ is the number of cells. In this model framework it is assumed that

$$
\boldsymbol{b}_{\boldsymbol{i}} \sim N\left(0, \sigma_{b}^{2}\right), \epsilon_{\boldsymbol{i}} \sim N\left(0, \sigma^{2} \boldsymbol{I}\right)
$$

where $\sigma_{\mathrm{b}}^{2}$ and $\sigma^{2}$ are the within-cell and between-cell variance, respectively. $I$ denotes an identity matrix. The lme function from the nlme package [24] in the statistical software $\mathrm{R}$ was used to fit the models. The $t$-statistic and the corresponding $p$-value for the slope in this model should be identical to the values obtained from a comparison of the two acquisitions using a paired $t$-test [25]. We verified this for all the models in this study by performing paired $t$-tests using the t.test function in $\mathrm{R}$, and comparing the results with the slope statistics in the output from the model-fitting using the lme function. The model given by Equation (4) can thus be used as a comparison of the two acquisitions. As described by Zuur et al. [22], assumptions about spatial autocorrelation between subjects can be introduced in a linear mixed effects model by replacing $I$ with a matrix $V$, such that

$$
\epsilon_{i} \sim N\left(0, \sigma^{2} V\right)
$$

with $V$ depending on the given correlation structure. Since the data from the two acquisitions could exhibit spatially-dependent variation, we tested if incorporating assumptions about spatial autocorrelation led to models which differed from the models without such assumptions. 
We fitted separate models with spherical and Gaussian correlation structures [23]. Each of these models was then compared to the model with assumed uncorrelated errors, given by Equations (4) and (5). Since this model is nested within the models with assumptions about correlated errors, a likelihood ratio test could be used for the comparisons [23].

\section{Results}

\subsection{Regression Models}

Two linear regression models were fit to the modeling dataset (Equations (1) and (2)). Both models showed good fit, with $\mathrm{R}^{2}$ values of 0.86 and 0.72 (Table 4 and Figure 5). A leave-one-out cross-validation of the two regression models resulted in RMSE\% of $28.5 \%$ and $44.7 \%$ for the height model (Equation (2)) and crown diameter model (Equation (1)), respectively (Table 4).
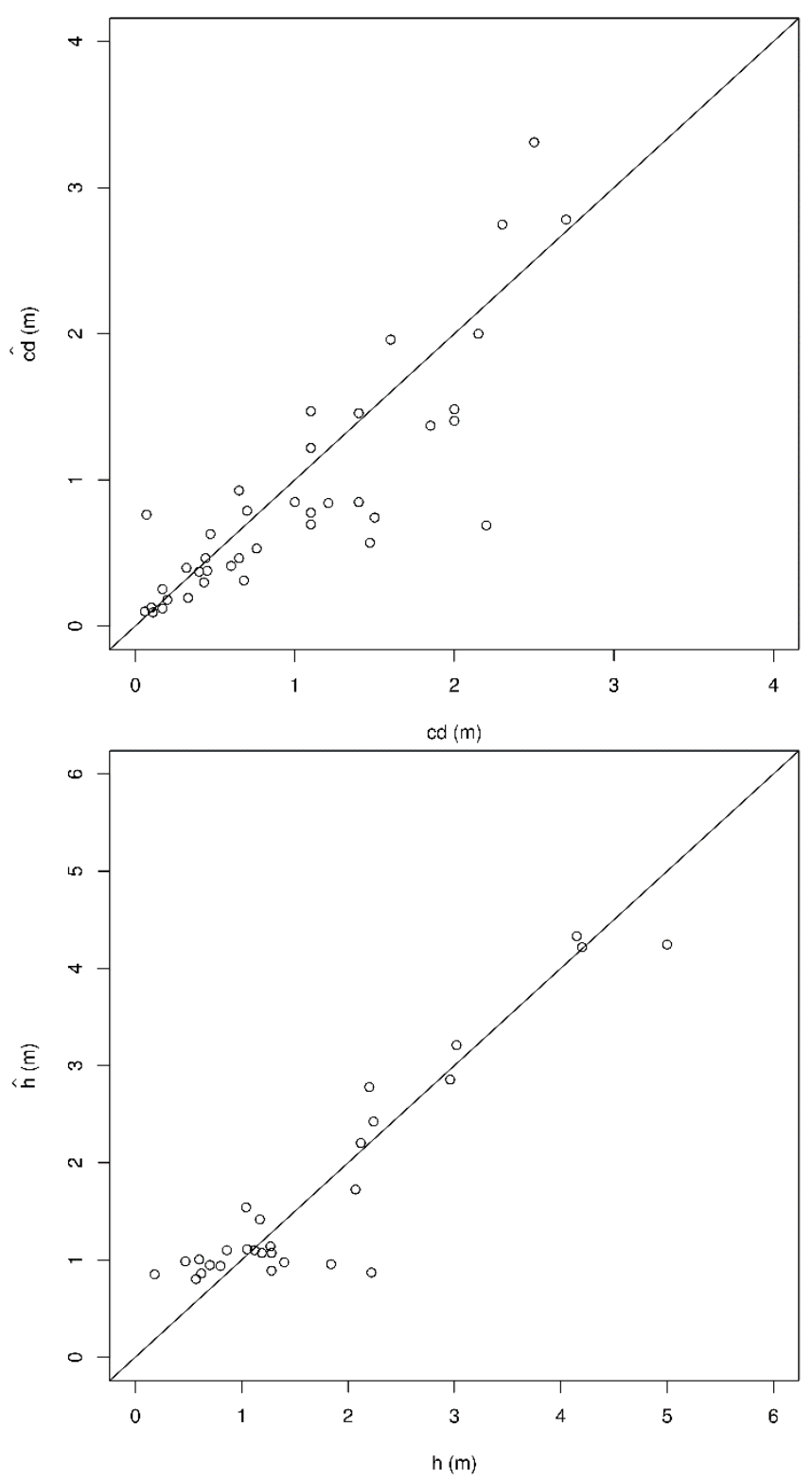

Figure 5. Observed versus predicted values for the two linear models: Height-crown diameter model given in Equation (1) (top), and ALS height-field height given in Equation (2) (bottom). 
Table 4. Regression model variables, parameter values and goodness of fit. RMSE from leave-on-out cross-validation.

\begin{tabular}{cccccc}
\hline Dependent Variable & Independent Variable & \multicolumn{2}{c}{ Parameter Values ${ }^{\text {a }}$} & $\boldsymbol{R}^{\mathbf{2}}$ & RMSE (RMSE\%) $^{\text {R }}$ \\
\hline$h$ & $h \max _{A L S}$ & $\beta_{b 0}: 0.8030$ & $\beta_{b 1}: 0.9590$ & 0.86 & $0.49(28.5)$ \\
$c d$ & $h$ & $\beta_{a}: 0.6621$ & & 0.72 & $0.45(44.7)$ \\
\hline
\end{tabular}

a Significance level for all parameters: $p<0.000$.

\subsection{Detection and Segmentation}

Following the procedure described in Section 2.4, individual crown segments were formed from the ALS echoes for the entire study area (Figures 6 and 7). We report results for the segmentation procedure with parameter $s=0.2$. Other values of $s$ gave only minor changes in the results, and this is further discussed in Section 4. The ALS-derived segments were compared to the field-measured crown segments (Figure 4). A successful match, i.e., a correctly segmented tree, was registered using the criteria given in Section 2.4. Overall, $46.2 \%$ of the trees were successfully segmented. Detection rates ranged from $15.8 \%$ to $80.4 \%$ for the individual height classes (see Section 2.2), with the detection rate increasing with tree size (Table 5).

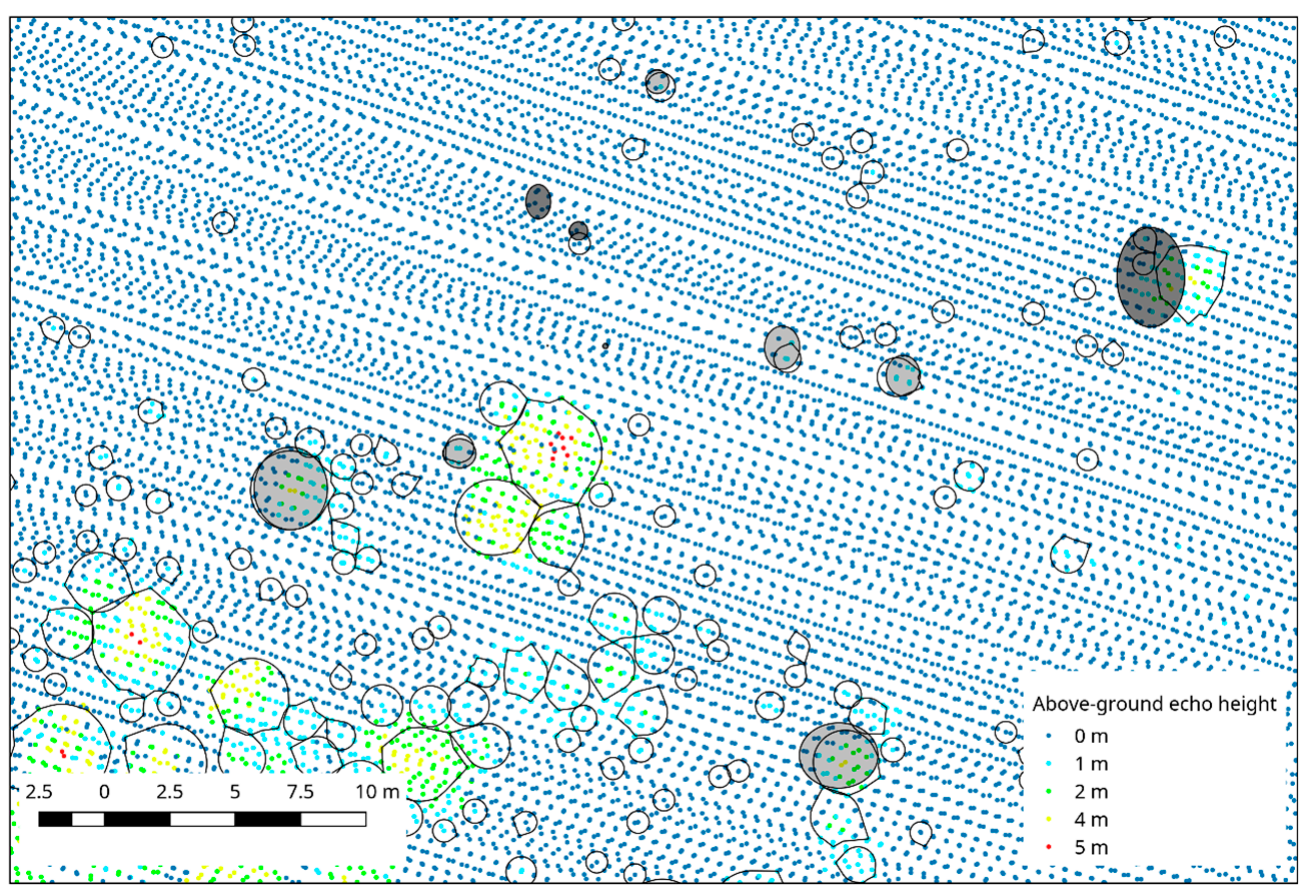

Figure 6. Single-tree segments from the described procedure (hollow segments) and field-measured crown ellipses of detected (light grey) and undetected (dark grey) trees. Note that only a sample of the trees was measured in the field. ALS echoes are colored according to the above-ground height.

Table 5. Detection rates with field-measured segments as reference.

\begin{tabular}{cc}
\hline Height Class & Correctly Segmented Trees (\%) \\
\hline $0-1 \mathrm{~m}$ & 15.8 \\
$1-2 \mathrm{~m}$ & 68.1 \\
$2-3 \mathrm{~m}$ & 75.5 \\
$>3 \mathrm{~m}$ & 80.4 \\
All & 46.2 \\
\hline
\end{tabular}




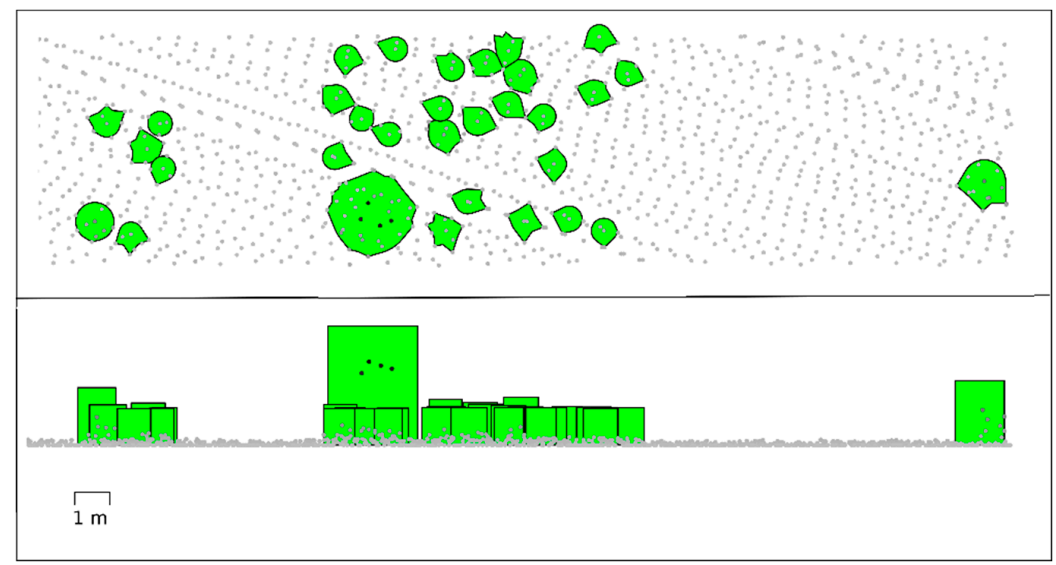

Figure 7. Visualization of single-tree segments and ALS echoes from ALS2012, covering the same area as Figure 2. Viewed from above (upper figure) and from the side (lower figure). The segments extent and estimated tree heights are colored green, and the ALS echoes are colored from grey to black. The highest echoes are colored black. Note that the terrain height has been subtracted from the ALS echo heights (see Section 2.3 for details).

\subsection{Stability}

Comparison of the linear mixed effects models with and without assumptions about spatial autocorrelation revealed that no differences could be found between the models, with $p$-values for the likelihood ratio tests ranging from 0.15 to 0.99 . This suggests that spatial autocorrelation does not have a major influence in this case, and that using an ordinary paired $t$-test is sufficient for testing the differences between the number of trees derived from the two acquisitions in each $200 \mathrm{~m}^{2}$ cell.

These tests for differences between the mean number of segmented trees from the two acquisitions resulted in $p$-values ranging from 0.27 to 0.65 , and it is evident from this result that no significant differences could be detected through these tests. It should be noted that this range included $p$-values from all comparisons, for the slope coefficients in the mixed models in which assumptions about spatial autocorrelation were included, as well as ordinary paired $t$-tests. Overall, this shows that for the 569 cells—with a total area of 11.4 ha-there were no significant differences between the mean number of segmented trees derived from the two same-day ALS acquisitions.

Some variation between the number of segmented trees from the two acquisitions was, however, observed, with the highest variation for the smaller trees (Table 6). Overall, the mean number of segmented trees in each cell was 52.0 and 51.7 for the two acquisitions. The mean of the differences between the number of segmented trees in each cell for the two acquisitions was 0.31 with a standard deviation of 10.20 (Table 6). It is, from this, evident that the number of segmented trees varies for individual cells. This variation is smaller if one considers only larger trees (Table 6).

Table 6. Comparison of the number of segmented trees within each $200 \mathrm{~m}^{2}$ cell derived using two separate ALS acquisitions (569 cells of $200 \mathrm{~m}^{2}$ ). Mean difference between the two acquisitions and the standard deviation for the difference (sd).

\begin{tabular}{ccccc}
\hline \multirow{2}{*}{ Height Class } & \multicolumn{3}{c}{ Number of Trees } \\
\cline { 2 - 5 } & Acquisition 1 & Acquisition 2 & \multicolumn{2}{c}{ Difference $^{\text {a }}$} \\
\cline { 2 - 5 } & Min-Max (Mean) & Min-Max (Mean) & Mean & sd \\
\hline $0-1 \mathrm{~m}$ & $0-139(37.8)$ & $0-170(37.7)$ & -0.38 & 10.04 \\
$1-2 \mathrm{~m}$ & $0-30(4.6)$ & $0-28(4.5)$ & -0.11 & 2.21 \\
$2-3 \mathrm{~m}$ & $0-30(3.7)$ & $0-32(3.8)$ & 0.08 & 1.82 \\
$>3 \mathrm{~m}$ & $0-31(6.3)$ & $0-30(6.3)$ & 0.04 & 1.27 \\
All & $1-147(51.7)$ & $1-181(52.0)$ & 0.31 & 10.20 \\
\hline
\end{tabular}

${ }^{a}$ No significant differences were observed, see text for details. 


\section{Discussion}

Over all the four height classes, $46.2 \%$ of the trees were correctly segmented by the proposed method. A higher proportion of the larger trees were correctly segmented and the detection rate decreased with the decreasing tree size. There are few directly comparable studies, since previous studies typically targeted trees that are larger than in the present study. Vauhkonen et al. [21] compared six single-tree detection algorithms in different types of mature forests. The average detection rates for the different forest types reported by Vauhkonen et al. varied between $54 \%$ and $91 \%$. These detection rates were, however, calculated using the plot-wise total number of tree segments in relation to the number of field-measured trees. They cannot be directly compared to the detection rates in the present study, which were calculated based on the linking field and ALS-derived trees. Vauhkonen et al. report a corresponding number called "treetop candidates linked to field trees", and this varied between $42 \%$ and $60 \%$. The mean tree diameter at the different sites in the data material used by Vauhkonen et al. was $18.5-35.8 \mathrm{~cm}$, and the ALS point density was $1.5-30$ per $\mathrm{m}^{2}$. The detection rates observed for trees with $h<1 \mathrm{~m}$ in the present study were considerably lower than the rates reported by Vauhkonen et al. [21]. These trees are, however, much smaller than any tree in the data material used in that study. For the trees with $h>1 \mathrm{~m}$, the proportion of detected field trees in the present study seems to be within the range reported in Vauhkonen et al. [21], as well as in recent studies by Liu et al. [26], and Mongus and Zalik [27].

The advantage of the proposed method is that it is simple, and can be implemented using models developed from a limited number of sample trees. A disadvantage is the need for these sample trees, as well as the detection errors discussed in the following.

From the current study it is evident that direct detection of all individual trees by ALS is not possible when the properties of the ALS data are similar to those of the current study. Omission errors will occur, caused by two different factors: firstly, due to limitations in the data material itself; ALS can be viewed as measuring distances from the aircraft to the ground, with the distance to only some particular spots on the ground being acquired. In the present study the average density of laser pulses was eight pulses per $\mathrm{m}^{2}$, and given the footprint size together with the non-uniform spatial distribution of the laser pulses, this means that some trees will not be hit at all. The fraction of the trees that is not hit by any laser pulse will depend on a range of factors, such as the pulse density, the pulse footprint size and the degree of unevenness in the spatial distribution of the pulses on the ground. It is, however, clear that the size of a tree directly affects the probability for it to be hit by a laser pulse, so smaller trees are less likely to be hit than larger trees. The trees which are not hit by any laser pulses cannot be directly detected using the ALS data, and it is hence a definite limit to direct detection of individual trees inherent in the data material itself. There is, under such conditions, not enough information in the data material to directly detect all trees, and omission errors are unavoidable if all trees, even the smallest ones, are considered. The chance of being hit by a laser pulse increases with the tree size, but even an echo reflected from the tree is in itself not sufficient to ensure a successful detection. To be able to separate it from the surrounding terrain, the echo must have a positive above-ground height.

The second cause of omission errors is in the segmentation procedure, which in some cases will fail to produce a segment that matches that of the tree on the ground. This can be seen in Figure 6, at the rightmost field-measured tree. The ALS-derived segment is, in this case, not similar enough to be considered a correct segmentation. The reason can be measurement errors, neighboring trees or other factors affecting how the echoes are being reflected from that particular tree. Segments from multiple trees can also be erroneously merged, and thus lead to omissions. How the procedure merges segments is controlled by the $s$ parameter, and this is discussed later in this section.

All echoes with a positive above-ground height will, however, not be reflected from trees, which is one out of two types of commission errors. Objects such as rocks, hummocks and bushes may all result in positive above-ground heights, and thus result in falsely detected trees. The intensity value of the echoes could, however, hold some information that can be used to distinguish between trees and 
the surrounding terrain and vegetation, and some studies have found a positive contribution from the intensity values when classifying tree and non-tree echoes [7].

The second type of commission error is over-segmentation, which means detecting several trees from the echoes reflected from a single tree. Due to the design of the field work in which only a sample of the trees was measured, we were not able to fully assess these commission errors. The parameter $s$ controls how the segmentation procedure merges initially overlapping segments, and the number of segments will increase as $s$ increases. So with $s=1$ all initial segments are kept as separate segments, whereas with $s=0$ all overlapping segments are merged, i.e., no final segments overlap. Since tree crowns sometimes do overlap, a reasonable value of $s$ should be somewhere between the two extremes. The detection rates in the present study varied, however, very little for the different tested values of $s$. This can be attributed to several factors, first of all that changing the value of $s$ will only affect segments that initially overlapped. Furthermore, it is clear that the presence of small segments at the edge of larger segments does not have a large influence on either detection or omission errors. So even with some small segments overlapping, the larger segment will still be connected with the field tree. These small segments will, on the other hand, directly influence the commission errors, and this is the type of error we were unable to control in the present study. Further research is needed in order to find an optimal value for $s$, and to fully assess the commission errors. It should be noted that the study area, as well as other transition zones between forest and alpine areas, is, in parts, sparsely populated with trees. In more dense forests, a larger proportion of the trees will have overlapping crowns, which will affect the performance of the proposed segmentation procedure, as well as the optimal value of the $s$ parameter.

The initial processing of ALS data for most applications related to forests or trees involves a choice of algorithms and corresponding parameter values. In the current study, this involves the echo classification as well as the computation of the above-ground heights. This choice of algorithm and parameter values will most likely influence further use of the data in, for example, single tree detection. The widely used classification algorithm based on the principles described by Axelsson $[18,28]$ is used in the present study. This algorithm requires parameter values for "iteration angle" and "iteration distance". The effect of the iteration angle on echoes reflected from small trees in the forest-tundra ecotone was investigated by Næsset [3]. In that study, an increase in omission errors and a decrease in commission errors were observed when the iteration angle was increased from six to 12 degrees. A tree was, in that study, regarded as detected if it yielded at least one echo with a positive above-ground height, and the terms omission and commission error refer to that definition. A conclusive suggestion on an optimal iteration angle was, however, not given based on those results. The use of a model-chain as in the proposed algorithm will cause errors to propagate and add up through the chain. Errors in the allometric model given by Equation (2) will, for example, affect the results from applying the model given in Equation (1), and finally the resulting single-tree segments.

When evaluating single-tree detection algorithms, the obtained tree segments will deviate from the field measurements. The process of choosing and defining the detection criteria will inevitably involve subjectivity. The choice of detection criteria will affect the detection rates, and the effect of detection criteria should be incorporated in the evaluation of segmentation algorithms.

In the case of a change assessment in which an identical detection method is applied at two points in time, omission and commission errors should theoretically be of less consequence. Given that these errors occur with the same magnitude in each of the two segmentations, actual changes on the ground between two ALS acquisitions should lead to corresponding differences in the two sets of segmented trees. The stability of the ALS-derived variables plays a role in this case. The amount of variation that is due to the scanning and the segmentation process itself will determine the magnitude of the vegetation changes that can be reliably detected for a given area. The results from the present study indicate that for the proposed segmentation procedure, some variation must be expected for smaller areas. The magnitude of the changes that can be reliably detected using the proposed method could be further investigated. The spatial distribution of the laser echoes on the ground and in the 
vegetation will differ from one acquisition to another. We assessed, in the present study, the influence of these differences on the resulting single-tree segments by using two separate acquisitions from the same sensor. The use of different sensors in multi-temporal data acquisition will further contribute to differences between the two sets of data. Expected effects and possible calibration methods to mitigate these could be subject to further research.

\section{Conclusions}

Moderate detection rates were observed when using the proposed segmentation algorithm. Overall, $46.2 \%$ of the trees were segmented correctly. The detection rates were higher for larger trees, and conversely, lower for smaller trees. The high proportion of undetected trees was partly due to limitations in the data material itself; some trees were not hit by any laser pulses at all. No significant differences between the number of segmented trees derived from two separate ALS acquisitions were found in the present study. This indicates that it can be suitable for monitoring purposes. Even though the magnitude of the detection errors prevents the detection of changes at an individual tree level, the method might potentially be used to detect changes at an area level. The use of the proposed method for area-based monitoring and change detection in the forest-tundra ecotone could be subject to further research. The proposed method could also be suitable for detection and monitoring of small trees in other biomes, such as seedlings in boreal forest or regeneration in tropical forests. This could be further investigated.

Acknowledgments: We wish to thank Eirik Næsset Ramtvedt for participating in the field work. Terratec AS and Blom ASA acquired and pre-processed the ALS data. We will also like to thank the anonymous reviewers for valuable comments and suggestions. The project was funded by the Norwegian Research Council through grant \#184636/S30.

Author Contributions: E.N. planned the acquisition of ALS data, planned and conducted the acquisition of field data and revised the manuscript. M.H. conceived and conducted the study and wrote the paper.

Conflicts of Interest: The authors declare no conflict of interest.

\section{Abbreviations}

The following abbreviations are used in this manuscript:

$\begin{array}{ll}\text { ALS } & \text { Airborne laser scanning } \\ \text { TIN } & \text { Triangular irregular network } \\ \text { RMSE } & \text { Root mean squared error } \\ \text { sd } & \text { Standard deviation }\end{array}$

\section{References}

1. Holtmeier, F.-K.; Broll, G. Treeline advance-Driving processes and adverse factors. Landsc. Online 2007, 1, 1-32. [CrossRef]

2. $\quad$ De Wit, H.A.; Bryn, A.; Hofgaard, A.; Karstensen, J.; Kvalevåg, M.M.; Peters, G.P. Climate warming feedback from mountain birch forest expansion: Reduced albedo dominates carbon uptake. Glob. Chang. Biol. 2014, 20, 2344-2355. [CrossRef] [PubMed]

3. Næsset, E. Influence of terrain model smoothing and flight and sensor configurations on detection of small pioneer trees in the boreal-alpine transition zone utilizing height metrics derived from airborne scanning lasers. Remote Sens. Environ. 2009, 113, 2210-2223. [CrossRef]

4. Næsset, E.; Nelson, R. Using airborne laser scanning to monitor tree migration in the boreal-alpine transition zone. Remote Sens. Environ. 2007, 110, 357-369. [CrossRef]

5. Reese, H.; Nystrom, M.; Nordkvist, K.; Olsson, H. Combining airborne laser scanning data and optical satellite data for classification of alpine vegetation. Int. J. Appl. Earth Obs. Geoinf. 2014, 27, 81-90. [CrossRef]

6. Rees, W.G. Characterisation of Arctic treelines by LiDAR and multispectral imagery. Polar Rec. 2007, 43, 345-352. [CrossRef] 
7. Stumberg, N.; Ørka, H.O.; Bollandsås, O.M.; Gobakken, T.; Næsset, E. Classifying tree and nontree echoes from airborne laser scanning in the forest-tundra ecotone. Can. J. Remote Sens. 2012, 38, 655-666. [CrossRef]

8. Thieme, N.; Martin Bollandsås, O.; Gobakken, T.; Næsset, E. Detection of small single trees in the forest-tundra ecotone using height values from airborne laser scanning. Can. J. Remote Sens. 2011, 37, 264-274. [CrossRef]

9. Chang, A.; Eo, Y.; Kim, Y.; Kim, Y. Identification of individual tree crowns from LiDAR data using a circle fitting algorithm with local maxima and minima filtering. Remote Sens. Lett. 2013, 4, 29-37. [CrossRef]

10. Hyyppä, J.; Kelle, O.; Lehikoinen, M.; Inkinen, M. A segmentation-based method to retrieve stem volume estimates from 3-D tree height models produced by laser scanners. IEEE Trans. Geosci. Remote Sens. 2001, 39, 969-975. [CrossRef]

11. Persson, A.; Holmgren, J.; Söderman, U. Detecting and measuring individual trees using an airborne laser scanner. Photogramm. Eng. Remote Sens. 2002, 68, 925-932.

12. Reitberger, J.; Schnörr, C.; Krzystek, P.; Stilla, U. 3D segmentation of single trees exploiting full waveform LIDAR data. ISPRS J. Photogramm. Remote Sens. 2009, 64, 561-574. [CrossRef]

13. Solberg, S.; Næsset, E.; Bollandsås, O.M. Single tree segmentation using airborne laser scanner data in a structurally heterogeneous spruce forest. Photogramm. Eng. Remote Sens. 2006, 72, 1369-1378. [CrossRef]

14. Koch, B.; Kattenborn, T.; Straub, C.; Vauhkonen, J. Segmentation of Forest to Tree Objects. In Forestry Applications of Airborne Laser Scanning; Maltamo, M., Næsset, E., Vauhkonen, J., Eds.; Springer: Dordrecht, The Netherlands, 2014; Volume 27, pp. 89-112.

15. Cottam, G.; Curtis, J.T. The use of distance measures in phytosociological sampling. Ecology 1956, 37, 451-460. [CrossRef]

16. Ussyshkin, R.V.; Smith, B. Performance analysis of ALTM 3100EA: Instrument specifications and accuracy of LiDAR data. In Proceedings of the ISPRS Conference, Commission I Symposium, Paris, France, 4-6 May 2006.

17. Leica ALS70-Airborne Laser Scanners-Performance for Diverse Applications. Available online: http:/ / leica-geosystems.com/products/airborne-systems/lidar/leica-als70-airborne-laser-scanner (accessed on 10 December 2015).

18. Axelsson, P. DEM generation from laser scanner data using adaptive TIN models. Int. Arch. Photogramm. Remote Sens. 2000, 33, 110-117.

19. Terrasolid Ltd. TerraScan User's Guide; Terrasolid Ltd.: Helsinki, Finland, 2011.

20. R Development Core Team. R: A Language and Environment for Statistical Computing; R Foundation for Statistical Computing: Vienna, Austria, 2011.

21. Vauhkonen, J.; Ene, L.; Gupta, S.; Heinzel, J.; Holmgren, J.; Pitkanen, J.; Solberg, S.; Wang, Y.; Weinacker, H.; Hauglin, K.M.; et al. Comparative testing of single-tree detection algorithms under different types of forest. Forestry 2012, 85, 27-40. [CrossRef]

22. Zuur, A.F.; Ieno, E.N.; Walker, N.; Saveliev, A.A.; Smith, G.M. Mixed Effects Models and Extensions in Ecology with R; Statistics for Biology and Health; Springer New York: New York, NY, USA, 2009.

23. Pinheiro, J.; Bates, D. Mixed-Effects Models in S and S-PLUS; Springer: New York, NY, USA, 2013.

24. Pinheiro, J.; Bates, D.; DebRoy, S.; Sarkar, D.; R Core Team. NLME: Linear and Nonlinear Mixed Effects Models. Available online: https:/ /CRAN.R-project.org/package=nlme (accessed on 3 March 2016).

25. Nyberg, J.S. The Paired T-Test: Does PROC MIXED Produce the Same Results as PROC TTEST? In Proceedings of the PharmaSUG Conference, San Diego, CA, USA, 23-26 May 2004; Volume 5.

26. Liu, T.; Im, J.; Quackenbush, L.J. A novel transferable individual tree crown delineation model based on Fishing Net Dragging and boundary classification. ISPRS-J. Photogramm. Remote Sens. 2015, 110, $34-47$. [CrossRef]

27. Mongus, D.; Žalik, B. An efficient approach to 3D single tree-crown delineation in LiDAR data. ISPRS J. Photogramm. Remote Sens. 2015, 108, 219-233. [CrossRef]

28. Axelsson, P. Processing of laser scanner data-Algorithms and applications. ISPRS J. Photogramm. Remote Sens. 1999, 54, 138-147. [CrossRef]

(C) 2016 by the authors; licensee MDPI, Basel, Switzerland. This article is an open access article distributed under the terms and conditions of the Creative Commons Attribution (CC-BY) license (http://creativecommons.org/licenses/by/4.0/). 